\title{
Performance Comparison of Doppler Scale Estimation Methods for Underwater Acoustic OFDM
}

\author{
Lei Wan, ${ }^{1}$ Zhaohui Wang, ${ }^{1}$ Shengli Zhou, ${ }^{1}$ T. C. Yang, ${ }^{2}$ and Zhijie Shi ${ }^{3}$ \\ ${ }^{1}$ Department of Electrical and Computer Engineering, University of Connecticut, 371 Fairfield Way U-2157, Storrs, CT 06269, USA \\ ${ }^{2}$ National Sun Yat-Sen University, Kaohsiung, Taiwan \\ ${ }^{3}$ Department of Computer Science and Engineering, University of Connecticut, 371 Fairfield Way U-2155, Storrs, CT 06269, USA
}

Correspondence should be addressed to Shengli Zhou, shengli@engr.uconn.edu

Received 13 January 2012; Accepted 5 April 2012

Academic Editor: Charalampos C. Tsimenidis

Copyright (C) 2012 Lei Wan et al. This is an open access article distributed under the Creative Commons Attribution License, which permits unrestricted use, distribution, and reproduction in any medium, provided the original work is properly cited.

Doppler scale estimation is one critical step needed by the resampling operation in acoustic communication receivers. In this paper, we compare different Doppler scale estimation methods using either cyclic-prefixed (CP) or zero-padded (ZP) orthogonalfrequency division-multiplexing (OFDM) waveforms. For a CP-OFDM preamble, a self-correlation method allows for blind Doppler scale estimation based on an embedded repetition structure while a cross-correlation method is available with the knowledge of the waveform. For each received ZP-OFDM block, the existence of null subcarriers allows for blind Doppler scale estimation. In addition, a pilot-aided method and a decision-aided method are applicable based on cross-correlation with templates constructed from symbols on pilot subcarriers only and from symbols on all subcarriers after data decoding, respectively. This paper carries out extensive comparisons among these methods using both simulated and real experimental data. Further, the applicabilities of these methods to distributed multiuser systems are investigated.

\section{Introduction}

Underwater acoustic communications and networking have been under extensive investigation in recent years $[1,2]$. Considerable progress on the physical layer communication techniques has been made for both single-carrier and multicarrier communications; see, for example, [3-19]. Relative to the radio channel which has relative short delay spread and slow time variation, underwater acoustic channels typically exhibit long delay spread and fast time variation. The latter brings significant Doppler effects to underwater acoustic communication systems, hence estimation of the Doppler scaling factor is one key receiver module [4, 20,21].

Typically, Doppler scale estimation is accomplished by inserting waveforms known to the receiver during the data transmission. Two popular approaches are described in the following.

(i) One approach is to use a pulse train which is formed by the repetition of a Doppler-insensitive waveform [22], such as linear-frequency-modulated
(LFM) waveform [23] and hyperbolic-frequencymodulated (HFM) waveform [24]. A transmission format with one preamble and one postamble around the data burst is usually adopted $[4,20,25]$, as shown in Figure 1. At the receiver side, by detecting the times-of-arrival of the preamble and postamble, thus the interval change in-between, an average Doppler scale estimate over the whole data burst can be obtained. Thanks to the Doppler-insensitive property of the waveforms, a single-branch-matched filtering operation is adequate even in the presence of Doppler distortion. However, this method is only suitable for offline processing due to the processing delay.

(ii) The other approach is to use a Doppler-sensitive waveform with a thumb-tack ambiguity function. A Doppler-sensitive waveform is usually transmitted as a preamble prior to the data burst, as shown in Figure 1. At the receiver side, a bank of correlators correlates the received signal with preambles prescaled by different Doppler scaling factors, and the branch with the largest correlation peak provides 


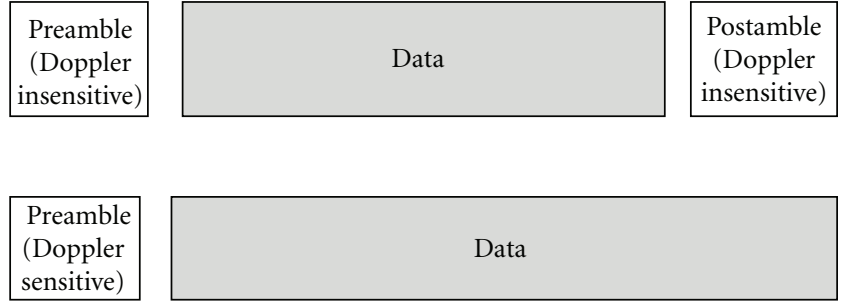

Figure 1: Top: Doppler scale estimation with Doppler-insensitive waveforms. Bottom: Doppler scale estimation with Dopplersensitive waveforms.

the estimated Doppler scale [25]. Typical Dopplersensitive waveforms include Costa waveforms [26], $\mathrm{m}$-sequence [27], and poly-phase sequence [28].

In this paper, we focus on an underwater acoustic communication system using zero-padded orthogonal-frequency division-multiplexing modulation (ZP-OFDM), in which pilot subcarriers and null subcarriers are usually multiplexed with data subcarriers for channel estimation and residual Doppler shift mitigation, respectively [4]. A cyclic prefixed (CP) OFDM preamble is inserted prior to data transmission for detection, synchronization, and Doppler scale estimation [29]. This transmission format, as shown in Figure 2, has been implemented on DSP-based OFDM modem prototypes [30].

By exploiting the cyclic repetition structure of the CPOFDM preamble, a blind estimation with a bank of selfcorrelators was proposed in [29]. However, it does not leverage the knowledge of the waveform itself which is known to the receiver. Taking this method as the first approach, one can easily construct the following Doppler scale estimators for the OFDM transmission in Figure 2.

(i) Cross-correlation with the CP-OFDM Preamble: Given the Doppler sensitivity of the OFDM waveform, a bank of cross-correlators can use the prescaled versions of the CP-OFDM waveform as local replicas.

(ii) Pilot-Aided Method for Each ZP-OFDM Block: By taking the waveform constituted by the pilot-subcarrier components as a replica of the transmitted signal, the Doppler estimation method using a bank of crosscorrelators is directly applicable.

(iii) Null-Subcarrier Based Blind Estimation Method for Each ZP-OFDM Block: As an extension of the blind carrier frequency offset (CFO) estimation method [31], the receiver rescales the received waveform with different tentative Doppler scaling factors and uses the energy on the null subcarriers to find the best fit.

(iv) Decision-Aided Method for Each ZP-OFDM Block: Once a ZP-OFDM block is successfully decoded, the transmitted waveform corresponding to this block can be reconstructed at the receiver. Taking the reconstructed waveform as a local replica, the Doppler estimation method using a bank of correlators can be deployed to refine the Doppler scale estimation for

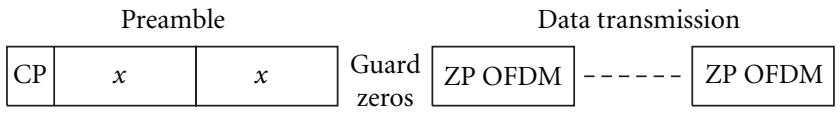

FIgURE 2: The data burst structure considered in this paper, which consists of a special CP-OFDM preamble and multiple ZP-OFDM blocks.

this block. The refined Doppler scale estimate can be passed to the next block.

The contributions of this paper are the following.

(i) We carry out extensive performance comparisons among the aforementioned Doppler estimation methods. Specifically, we focus on the OFDM transmission format in Figure 2 in single-user transmissions. Both simulations and experimental results reveal that the correlation-based methods have a decent performance in the low SNR region, and the blind estimation methods can catch up or even outperform the correlation methods in the high SNR region. As a performance benchmark, the CramerRao lower bound (CRLB) is also included for singlepath channels.

(ii) We extend our investigation to a multiuser OFDM setting, where different users could have different Doppler scaling factors [32]. Simulation results show that the correlation-based methods are robust to the multiuser interference, while the blind method suffers severe performance degradation.

The rest of this paper is as follows. Different Doppler scale estimation methods for CP-OFDM and ZP-OFDM waveforms are presented in Sections 2 and 3, respectively. Simulation results of these methods are provided in Section 4, and experimental results are provided in Section 5. Extension to the multiuser scenario is described in Section 6. Conclusions are contained in Section 7.

\section{Doppler Scale Estimation with a CP-OFDM Preamble}

Consider a CP-OFDM preamble structure in Figure 2, which consists of two identical OFDM symbols of length $T_{0}$ and a cyclic prefix of length $T_{\mathrm{cp}}$ in front, with the embedded structure

$$
x_{\mathrm{cp}}(t)=x_{\mathrm{cp}}\left(t+T_{0}\right), \quad-T_{\mathrm{cp}} \leq t \leq T_{0} .
$$

Let $B$ denote the system bandwidth, and define $K_{0}:=$ $B T_{0}$ as the number of subcarriers. The baseband CP-OFDM signal is

$$
x_{\mathrm{cp}}(t)=\sum_{k=-K_{0} / 2}^{K_{0} / 2-1} d[k] e^{j 2 \pi\left(k / 2 T_{0}\right)} q(t), \quad t \in\left[-T_{\mathrm{cp}}, 2 T_{0}\right],
$$


where $d[k]$ is the transmitted symbol on the $k$ th subcarrier, and $q(t)$ is a pulse shaping window,

$$
q(t)= \begin{cases}1, & t \in\left[-T_{\mathrm{cp}}, 2 T_{0}\right] . \\ 0, & \text { elsewhere. }\end{cases}
$$

The passband signal can be obtained as $\tilde{x}_{\mathrm{cp}}(t)=$ $2 \operatorname{Re}\left\{x_{\mathrm{cp}}(t) e^{j 2 \pi f_{\mathrm{c}} t}\right\}$, where $f_{\mathrm{c}}$ is the center frequency.

Consider a multipath channel which consists of $N_{\text {pa }}$ paths

$$
h(t ; \tau)=\sum_{p=1}^{N_{\mathrm{pa}}} A_{p}(t) \delta\left(t-\tau_{p}(t)\right),
$$

where $A_{p}(t)$ and $\tau_{p}(t)$ denote the amplitude and delay of the $p$ th path, respectively. Throughout this paper, we assume that the amplitude is constant within each OFDM block (about $200 \mathrm{~ms}$ for the system considered in this paper), that is, $A_{p}(t) \approx A_{p}$, which leads to

$$
h(t ; \tau)=\sum_{p=1}^{N_{\mathrm{pa}}} A_{p} \delta\left(t-\tau_{p}(t)\right) .
$$

After transmitting the passband signal $\tilde{x}_{\mathrm{cp}}(t)$ through the multipath channel, the received passband signal $\tilde{y}(t)$ is converted to baseband as $y(t)=\operatorname{LPF}\left(\tilde{y}(t) e^{-j 2 \pi f_{c} t}\right)$, where LPF denotes the low pass filtering operation.

2.1. Self-Correlation. If all the paths in the channel have the same Doppler scale factor

$$
\tau_{p}(t)=\tau_{p}-a t,
$$

it is shown in [29] that the embedded structure in the received waveform becomes

$$
\begin{aligned}
y(t)= & e^{-j 2 \pi(a /(1+a)) f_{\mathrm{c}} T_{0}} y\left(t+\frac{T_{0}}{1+a}\right), \\
& -\frac{T_{\mathrm{cp}}-\tau_{\max }}{1+a} \leq t \leq \frac{T_{0}}{1+a},
\end{aligned}
$$

which has a repetition period $T_{0} /(1+a)$ regardless of the channel amplitudes.

By exploiting the structure in (7), the time-of-arrival and the Doppler scale of the CP-OFDM symbol in the received signal can be jointly estimated via

$$
(\hat{a}, \hat{\tau})=\arg \max _{a, \tau}\left|\int_{0}^{T_{0} /(1+a)} y(t+\tau) y^{*}\left(t+\tau+\frac{T_{0}}{1+a}\right) d t\right|,
$$

which does not require the knowledge of the channel and the data symbols. This method can be implemented with a bank of self-correlators [29].

2.2. Cross-Correlation. Rather than exploiting the structure of the CP-OFDM preamble, the cross-correlation-based method can be used, since the transmitted preamble is known at the receiver. Taking the basic unit of duration $T_{0}$ as the template, the joint time-of-arrival and Doppler rate estimation can be achieved via

$$
(\hat{a}, \hat{\tau})=\arg \max _{a, \tau}\left|\int_{0}^{T_{0}} y(t+\tau) x_{\mathrm{cp}}^{*}((1+a) t) e^{-j 2 \pi a f_{\mathrm{c}} t} d t\right| .
$$

This can be implemented via a bank of cross-correlators, where the branch yielding the largest peak provides the needed Doppler scale estimate.

\section{Doppler Scale Estimation with Each ZP-OFDM Block}

As described in [4], a ZP-OFDM signal design multiplexing pilot and null subcarriers with data subcarriers can effectively deal with fast channel variations. Assume that the ZP-OFDM system has $K$ subcarriers. Let $T$ denote the symbol duration and $T_{\mathrm{g}}$ the guard interval. The total OFDM block duration is thus $T_{\mathrm{bl}}:=T+T_{\mathrm{g}}$. Denote $\wp_{\mathrm{D}}, \wp_{\mathrm{P}}, \wp_{\mathrm{N}}$ as the nonoverlapped sets formed by the data subcarriers, pilot subcarriers, and null subcarriers, respectively, which satisfy $\wp_{\mathrm{D}} \bigcup \wp_{\mathrm{P}} \bigcup \wp_{\mathrm{N}}=$ $\{-K / 2, \ldots, K / 2-1\}$. The baseband transmitted ZP-OFDM signal can be expressed by

$$
x_{\mathrm{zp}}(t)=\sum_{k \in \wp_{\mathrm{D}} \bigcup \S_{\mathrm{P}}} d[k] e^{j 2 \pi(k / T) t} g(t), \quad t \in\left[0, T_{\mathrm{bl}}\right],
$$

where $g(t)$ describes the zero-padding operation, that is,

$$
g(t)= \begin{cases}1, & t \in[0, T] \\ 0, & \text { elsewhere }\end{cases}
$$

After transmitting the ZP-OFDM symbol through a multipath channel defined in (5), we denote $\tilde{y}(t)$ as the received passband signal, whose baseband version is $y(t)=$ $\operatorname{LPF}\left(\tilde{y}(t) e^{-j 2 \pi f_{c} t}\right)$. The availability of null subcarriers, pilot subcarriers, and data subcarriers can be used for Doppler scale estimation.

3.1. Null-Subcarrier-Based Blind Estimation. In [29], the null subcarriers in ZP-OFDM system are exploited to perform carrier frequency offset (CFO) estimation. Here in this paper, the same principle is used to estimate Doppler scale factor.

Assume that coarse synchronization is available from the preamble. After truncating each ZP-OFDM block from the received signal, we resample one block with different tentative scaling factors. The total energy of frequency measurements at null subcarriers are used as a metric for the Doppler scale estimation

$$
\hat{a}=\arg \min _{a} \sum_{k \in \delta_{\mathrm{N}}}\left|\int_{0}^{T+T_{\mathrm{g}}} y\left(\frac{t}{1+a}\right) e^{-j 2 \pi a f_{c} t} e^{-j 2 \pi(k / T) t} d t\right|^{2} .
$$

For each tentative $a$, a resampling operation is carried out followed by fast Fourier transform. A one-dimensional grid search leads to a Doppler scale estimate. 
3.2. Pilot-Aided Estimation. As introduced above, a set of subcarriers $\varsigma_{\mathrm{P}}$ is dedicated to transmit pilot symbols. Hence, the transmitted waveform $x_{\mathrm{zp}}(t)$ is partially known, containing

$$
x_{\text {pilot }}(t)=\sum_{k \in \oiint_{\mathrm{P}}} d[k] e^{j 2 \pi(k / T) t} g(t), \quad t \in[0, T] .
$$

The joint time-of-arrival and Doppler scale estimation is achieved via

$$
\begin{aligned}
& (\hat{a}, \hat{\tau}) \\
& =\arg \max _{a, \tau}\left|\int_{0}^{T /(1+a)} y(t+\tau) x_{\text {pilot }}^{*}((1+a) t-\tau) e^{-j 2 \pi a f_{\mathrm{c}} t} d t\right|,
\end{aligned}
$$

which can be implemented via a bank of cross-correlators.

3.3. Decision-Aided Estimation. For an OFDM transmission with multiple blocks, the Doppler estimated in one block can be used for the resampling operation of the next block assuming small Doppler variation across blocks. After the decoding operation the receiver can reconstruct the transmitted time-domain waveform, by replacing $d[k]$ by its estimate $\hat{d}[k]$, for all $k \in \varsigma_{\mathrm{D}}$ in. Denote the reconstructed waveform as $\hat{x}_{\mathrm{zp}}(t)$.

Similar to the pilot-aided method, the decision-aided method performs the joint time-of-arrival and Doppler scale estimation via

$$
\begin{aligned}
& (\hat{a}, \hat{\tau}) \\
& =\arg \max _{a, \tau}\left|\int_{0}^{T /(1+a)} y(t+\tau) \hat{x}_{\mathrm{zp}}^{*}((1+a) t-\tau) e^{-j 2 \pi a f_{\mathrm{c}} t} d t\right|,
\end{aligned}
$$

which again is implemented via a bank of cross-correlators. The estimated $\hat{a}$ can be used for the resampling operation of the next block.

Remark 1. Relative to the pilot-aided method, the decisionaided method leverages the estimated information symbols, thus is expected to achieve a better estimation performance. Assuming that all the information symbols have been successfully decoded, the decision-aided method has knowledge about both the data and pilot symbols. Let $\left|\wp_{\mathrm{P}}\right|$ and $\left|\wp_{\mathrm{D}}\right|$ denote the numbers of pilot and data symbols, respectively. Using the template $\hat{x}_{\mathrm{zp}}(t)$ constructed from $\left(\left|\wp_{\mathrm{P}}\right|+\left|\wp_{\mathrm{D}}\right|\right)$ known symbols for cross correlation achieve a $10 \log _{10}\left(\left(\left|\wp_{\mathrm{P}}\right|+\left|\wp_{\mathrm{D}}\right|\right) /\left|\wp_{\mathrm{P}}\right|\right) \mathrm{dB}$ power gain in terms of noise reduction, relative to that using the template $x_{\text {pilot }}(t)$ constructed from $\left|\wp_{\mathrm{P}}\right|$ known symbols.

\section{Simulation Results}

The OFDM parameters are summarized in Table 1. For CP-OFDM, the data symbols at all the 512 subcarriers are randomly drawn from a QPSK constellation. For ZP-OFDM, out of 1024 subcarriers, there are $\left|\delta_{\mathrm{N}}\right|=96$ null subcarriers with 24 on each edge of the signal band for band protection
TABLE 1: OFDM parameters in simulations.

\begin{tabular}{lcc}
\hline System parameters & CP-OFDM & ZP-OFDM \\
\hline Center frequency: $f_{\mathrm{c}}$ & $13 \mathrm{kHz}$ & $13 \mathrm{kHz}$ \\
Bandwidth: $B$ & $4.88 \mathrm{kHz}$ & $4.88 \mathrm{kHz}$ \\
\# of subcarriers: & $K_{0}=512$ & $K=1024$ \\
Time duration: & $T_{0}=104.86 \mathrm{~ms}$ & $T=209.72 \mathrm{~ms}$ \\
Guard interval: & $T_{\mathrm{cp}}=100 \mathrm{~ms}$ & $T_{\mathrm{g}}=40.3 \mathrm{~ms}$ \\
\hline
\end{tabular}

and 48 evenly distributed in the middle for the carrier frequency offset estimation; $\left|\wp_{\mathrm{P}}\right|=256$ are pilot subcarriers uniformly distributed among the 1024 subcarriers, and the remaining are $\left|\wp_{\mathrm{D}}\right|=672$ data subcarriers for delivering information symbols. The pilot symbols are drawn randomly from a QPSK constellation. The data symbols are encoded with a rate-1/2 nonbinary LDPC code [33] and modulated by a QPSK constellation.

Three UWA channel settings are tested.

(i) Channel Setting 1: A single-path channel:

$$
h(t, \tau)=\delta(t-[\tau-a t]) .
$$

(ii) Channel Setting 2: A multipath channel with $N_{\text {pa }}=$ 15 paths, where all paths have one common Doppler scaling factor:

$$
h(t, \tau)=\sum_{p=1}^{N_{\mathrm{pa}}} A_{p} \delta\left(t-\left[\tau_{p}-a t\right]\right) .
$$

(iii) Channel Setting 3: A multipath channel with $N_{\text {pa }}=$ 15 paths, where each path has an individual Doppler scaling factor:

$$
h(t, \tau)=\sum_{p=1}^{N_{\mathrm{pa}}} A_{p} \delta\left(t-\left[\tau_{p}-a_{p} t\right]\right) .
$$

The interarrival time of paths follows an exponential distribution with a mean of $1 \mathrm{~ms}$. The mean delay spread for the channels in and is thus $15 \mathrm{~ms}$. The amplitudes of paths are Rayleigh distributed with the average power decreasing exponentially with the delay, where the difference between the beginning and the end of the guard time is $20 \mathrm{~dB}$. For each path, the Doppler scale $a_{p}$ is generated from a Doppler speed $v_{p}$ (with unit $\left.\mathrm{m} / \mathrm{s}\right)$ :

$$
a_{p}=\frac{v_{p}}{c},
$$

where $c=1500 \mathrm{~m} / \mathrm{s}$ is the sound speed in water. In channel settings 1 and 2, the Doppler speed $v$ is uniformly distributed within $[-4.5,4.5] \mathrm{m} / \mathrm{s}$. In channel setting 3 , the Doppler speeds $\left\{v_{p}\right\}$ are randomly drawn from the interval [1.5 $0.1,1.5+0.1] \mathrm{m} / \mathrm{s}$. 


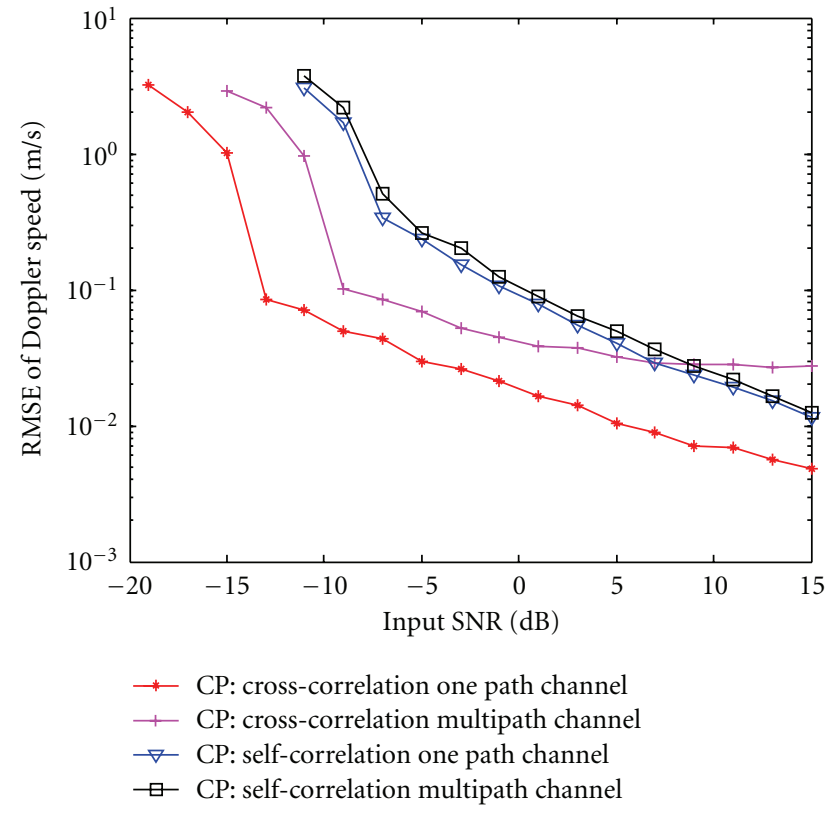

FIGURE 3: Performance of different estimators for the CP-OFDM preamble in single-path and multipath channels (channel settings 1 and 2).

In channel settings 1 and 2, the ground truths of $v$ and $a$ are known. We adopt the root-mean-squared-error (RMSE) of the estimated Doppler speed as the performance metric,

$$
\operatorname{RMSE}=\sqrt{E\left[|\hat{v}-v|^{2}\right]}=\sqrt{E\left[|(\hat{a}-a) c|^{2}\right]}
$$

which has the unit $\mathrm{m} / \mathrm{s}$. In channel setting 3, different paths have different Doppler scales, while the Doppler scale estimator only provides one estimate. RMSE is hence not well motivated. With the estimated Doppler scale to perform the resampling operation, we will use the block-error-rate (BLER) of the ZP-OFDM decoding as the performance metric.

4.1. RMSE Performance with CP-OFDM. For the singlepath channel, Figure 3 shows the RMSE performance of two estimation methods at different SNR levels. One can see a considerable gap between the self-correlation method and the cross-correlation method, while in the medium to high SNR region, both methods can provide a reasonable performance to facilitate receiver decoding.

For the multipath channel with a single Doppler speed, Figure 3 shows the RMSE performance of two estimation methods. One can see that the cross-correlation method outperforms the self-correlation method considerably in the low SNR region. However, the former suffers an error floor in the high SNR region, while the later does not.

Relative to the RMSE performance in the single-path channel, a considerable performance degradation can be observed for the cross-correlation method in the multipath channel, whereas the performance of the self-correlation method is quite robust. The reason for the difference lies in

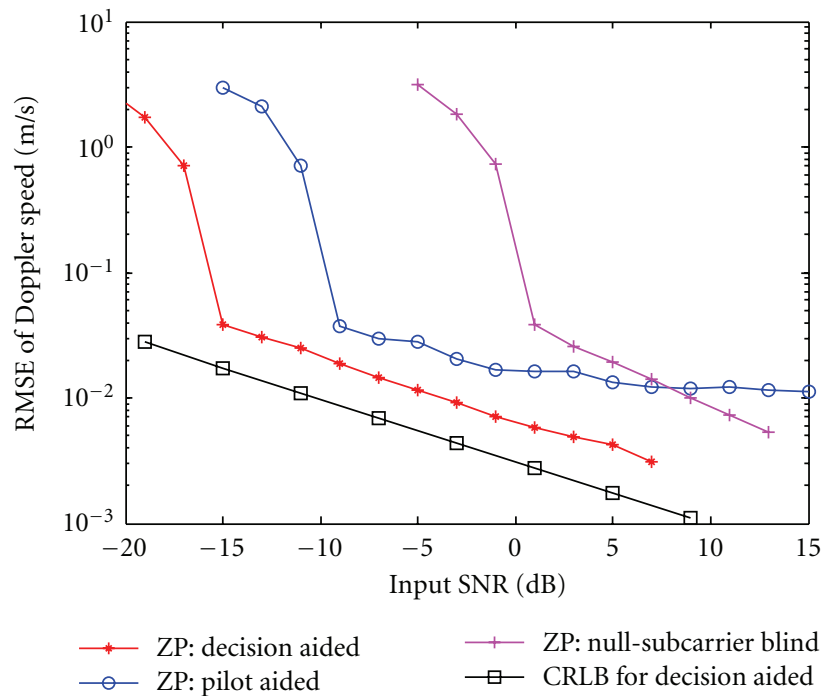

Figure 4: Performance of different estimators for ZP-OFDM in single-path channels (channel setting 1). The CRLB with all data known is included as a benchmark.

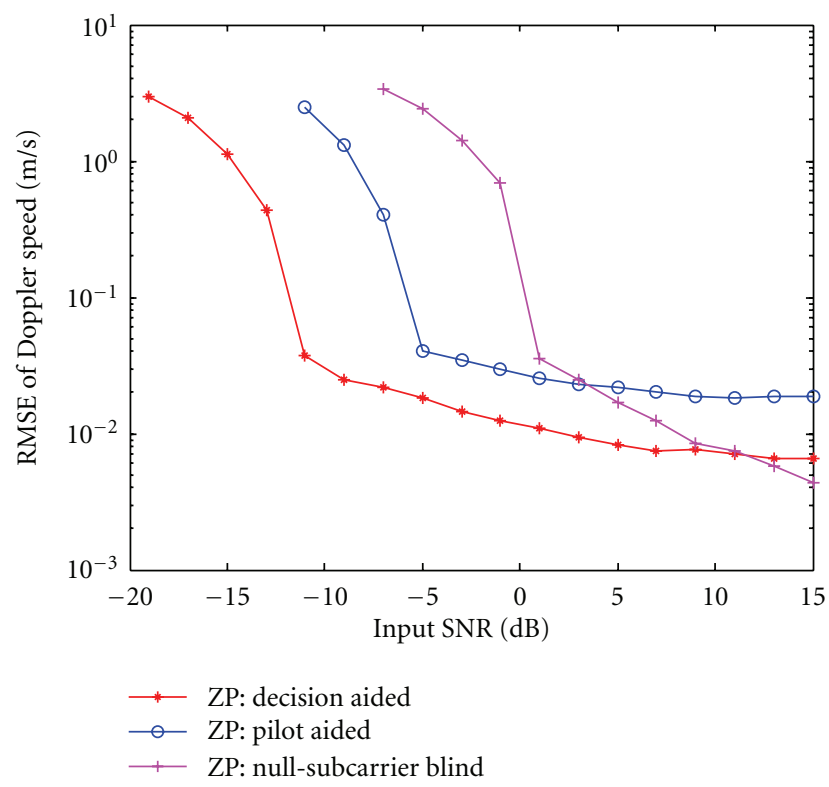

Figure 5: Performance of different estimators for ZP-OFDM in multipath channels with a common Doppler scale (channel setting 2).

the capability of the self-correlation method to collect the energy from all paths for Doppler scale estimation, while the cross-correlation method aims to get the Doppler scale estimate from only one path, the strongest path.

4.2. RMSE Performance with ZP-OFDM. Figure 4 shows the RMSE performance of three estimation methods for ZPOFDM in single-path channels. In the low SNR region, one can see that the decision-aided method is the best, while the null-subcarrier-based blind method is the worst. As discussed in Remark 1, the decision-aided method achieves 


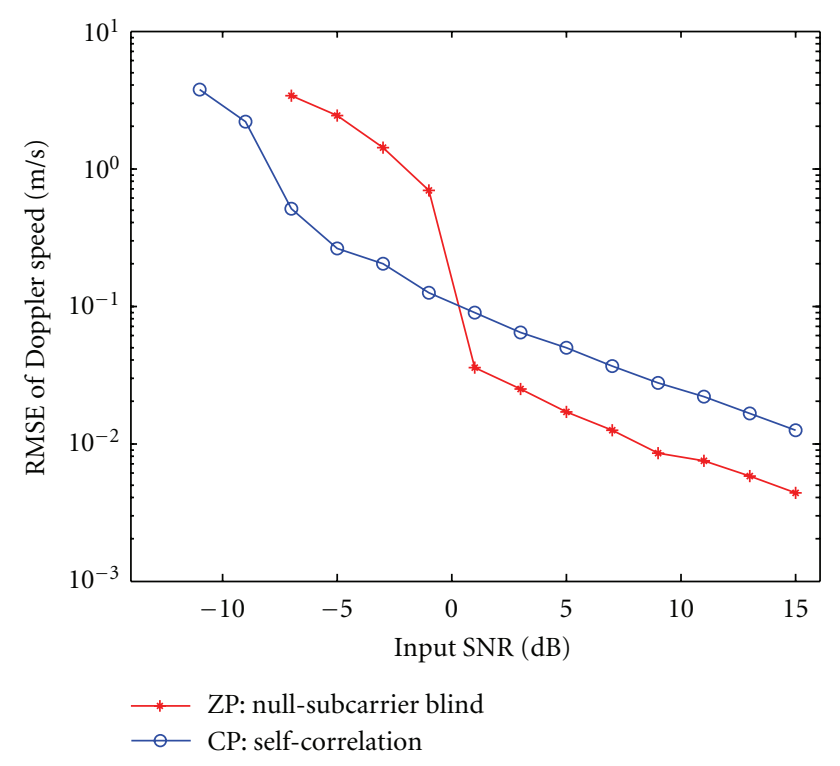

Figure 6: Null-subcarrier-based method in ZP-OFDM and CPOFDM.

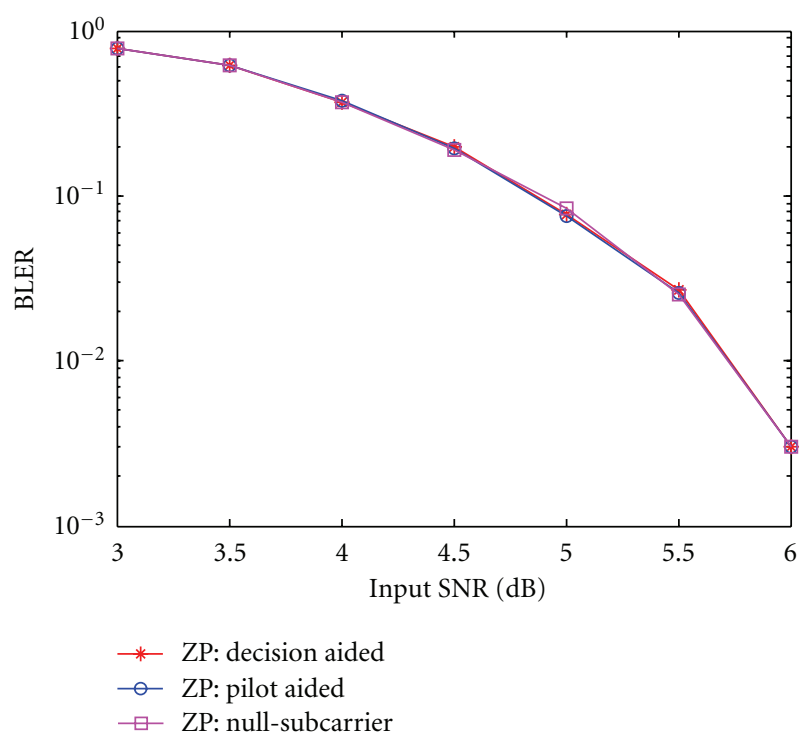

FIgURE 7: The BLER performance in multipath multi-Doppler channels (channel setting 3).

$\operatorname{10}_{10}\left(\left(\left|\wp_{\mathrm{D}}\right|+\left|\wp_{\mathrm{P}}\right|\right) /\left|\wp_{\mathrm{P}}\right|\right) \approx 6 \mathrm{~dB}$ power gain relative to the pilot-aided method. In the medium and high SNR region, the pilot-aided method suffers an error floor due to the interference from the data subcarriers, and the null-subcarrier-based blind method gets a good estimation performance. The Cramer-Rao lower bound (CRLB) with a known waveform is also included as the performance benchmark, whose derivation can be carried out similar to $[34,35]$.

Figure 5 shows the RMSE performance of three methods in multipath channels with a common Doppler speed. For each realization, the Doppler scale, the path amplitudes, and delays are randomly generated. The RMSE corresponding

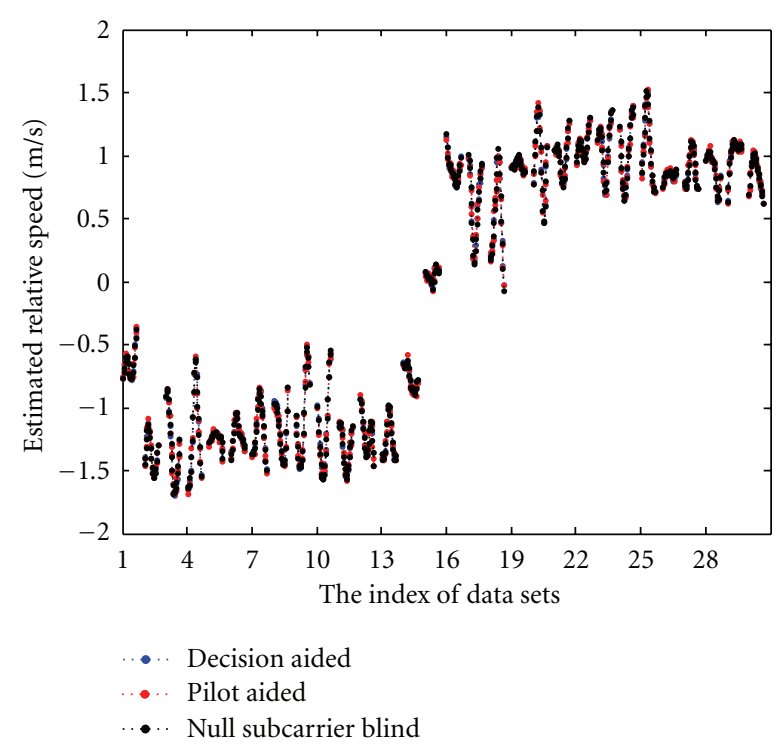

Figure 8: MACE10: Estimated Doppler speeds for 30 data bursts in MACE10, where each data burst has 20 OFDM blocks. The time interval between two consecutive date bursts is around 4 mins.

to each method is calculated by averaging the estimation error over multiple realizations. Again, one can see that in the low SNR region, the decision-aided method has the best performance, while the null-subcarrier-based blind method is the worst. Different from the performance in the singlepath channel, the decision-aided method has an error floor in the high SNR region, since it only picks up the maximum correlation peak of one path. On the other hand, the nullsubcarrier method has robust performance in the presence of multiple paths.

4.3. Comparison of Blind Methods of CP- and ZP-OFDM. The self-correlation method for the CP-OFDM preamble is closely related to the null-subcarrier-based blind method for ZP-OFDM. This can be easily verified by rewriting (2) as

$$
x_{\mathrm{cp}}(t)=\sum_{k=-K_{0}}^{K_{0}-1} d^{\prime}[k] e^{j 2 \pi\left(k /\left(2 T_{0}\right)\right) t} q(t), \quad t \in\left[-T_{\mathrm{cp}}, 2 T_{0}\right],
$$

where $d^{\prime}[k]=0$ when $k$ is odd and $d^{\prime}[k]=d[k / 2]$ when $k$ is even. The cyclic repetition pattern in is generated by placing zeros on all odd subcarriers in a long OFDM symbol of duration $2 T_{0}$. Hence, the self-correlation implementation could be replaced by the null-subcarrier-based implementation for the CP-OFDM preamble.

Figure 6 shows the performance comparison between the blind method for ZP-OFDM and that for CP-OFDM in the multipath channel with one Doppler scale factor, respectively. At low SNR, typically when it's lower than $0 \mathrm{~dB}$, the null-subcarrier-based method in CP-OFDM system has a better performance than that in the ZP-OFDM system, which is due to the fact that CP-OFDM system has 512 null subcarriers, more than 96 null subcarriers in the ZP-OFDM 


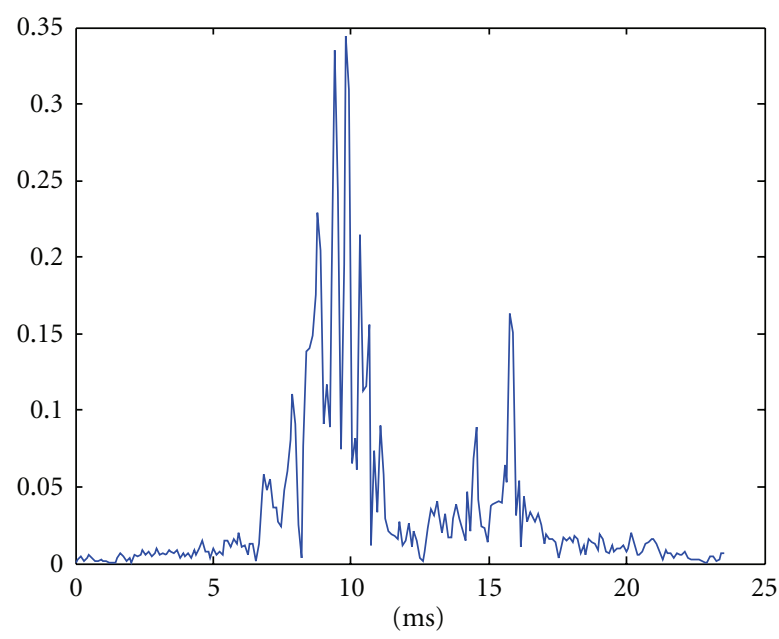

(a) File ID: 1750155F1978_C0_S5

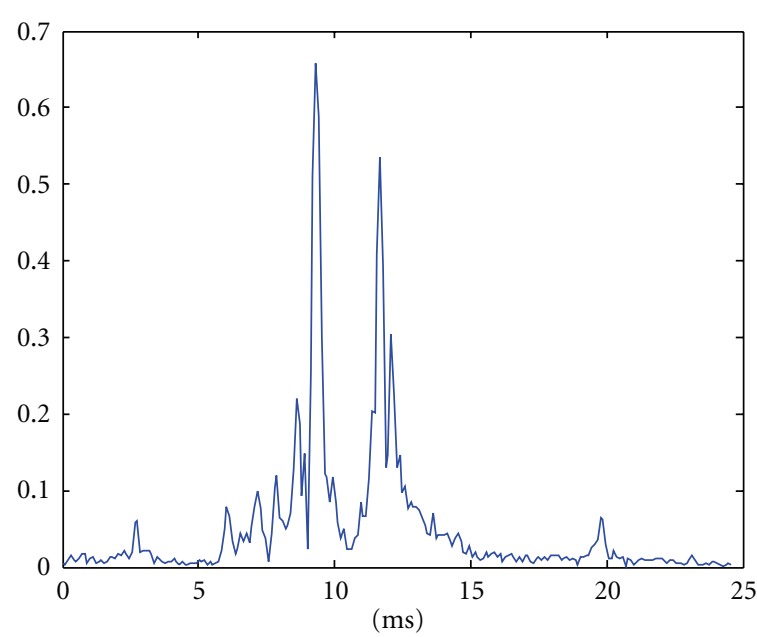

(b) File ID: 1750155F2070_C0_S5

FIGURE 9: Estimated channel impulse responses for two different blocks at different bursts.

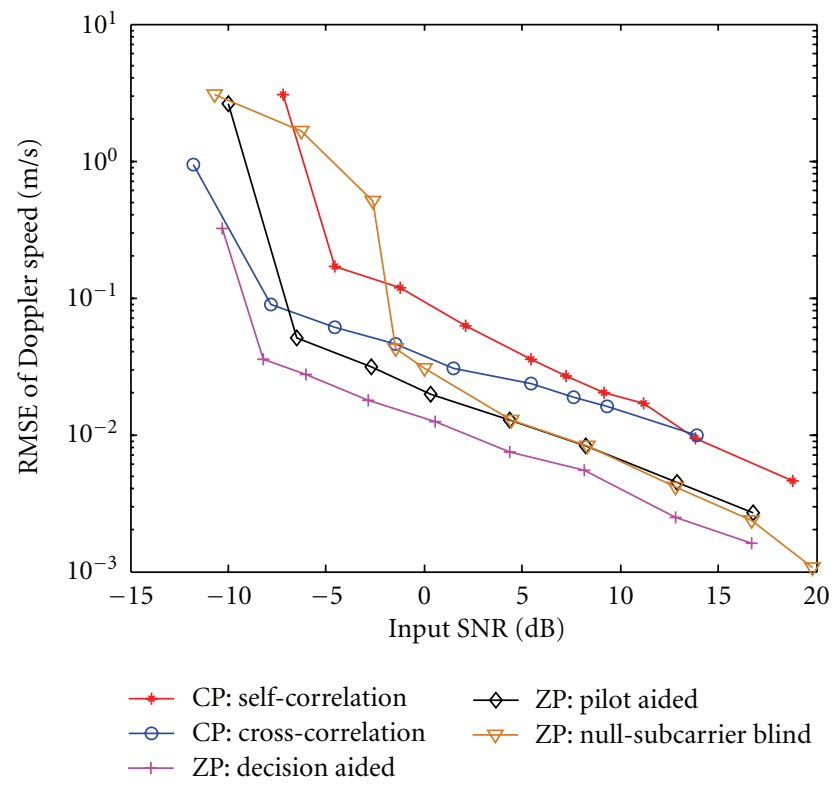

Figure 10: MACE10: Performance comparison of Doppler estimation approaches, file ID: 1750155F1954_C0_S5.

block. At high SNR, the null-subcarrier-based method in ZP-OFDM has better performance. The possible reason is that null subcarriers in ZP-OFDM are distributed with an irregular pattern, which could outperform the regular pattern in the CP-OFDM preamble.

4.4. BLER Performance with ZP-OFDM. With channels generated according to the channel setting 3 , Figure 7 shows the simulated BLER performance, where the received OFDM blocks are resampled with the Doppler scale estimates from different estimators and processed using the receiver from [4] and the LDPC decoder from [33]. At each SNR point, at least 20 block errors are collected.
It is expected that the OFDM system can only work when the useful signal power is above that of the ambient noise. Regarding the simulation results in Figure 5, one can see that all the methods can reach a RMSE lower than $0.1 \mathrm{~m} / \mathrm{s}$. Hence, it is not surprising that these methods lead to quite similar BLER results as shown in Figure 7. This observation is consistent with the analysis in [29] that an estimation error of $0.1 \mathrm{~m} / \mathrm{s}$ introduces a negligible error.

\section{Experimental Results}

This mobile acoustic communication experiment (MACE10) was carried out off the coast of Martha's Vineyard, Massachusetts, June, 2010. The water depth was about 80 meters. The receiving array was stationary, while the source was towed slowly away from the receiver and then towed back, at a speed around $1 \mathrm{~m} / \mathrm{s}$. The relative distance of the transmitter and the receiver changed from $500 \mathrm{~m}$ to $4.5 \mathrm{~km}$. Out of the two tows in this experiment, we only consider the data collected in the first tow. There are 31 transmissions in total, with a CP-OFDM preamble and 20 ZP-OFDM blocks in each transmission. We exclude one transmission file recorded during the turn of the source, where the SNR of the received signal is quite low.

The CP-OFDM and ZP-OFDM parameters and signal structures are identical to that in the simulation, as listed in Table 1.

Figure 8 shows the estimated Doppler speeds for ZPOFDM blocks from different methods. Clearly, the Doppler speed fluctuates from block to block. Figure 9 shows the estimated channel impulse responses for two ZP-OFDM blocks from two data sets, where the time interval between these two data bursts is more than 1 hour. The channels have a delay spread about $20 \mathrm{~ms}$ but with different delay profiles.

Based on the recorded files, we carried out two tests.

(A) Test Case 1. In this test, we focus on one single file (file ID: 1750155F1954_C0_S5) and compare the RMSE performance of different approaches by adding artificial 


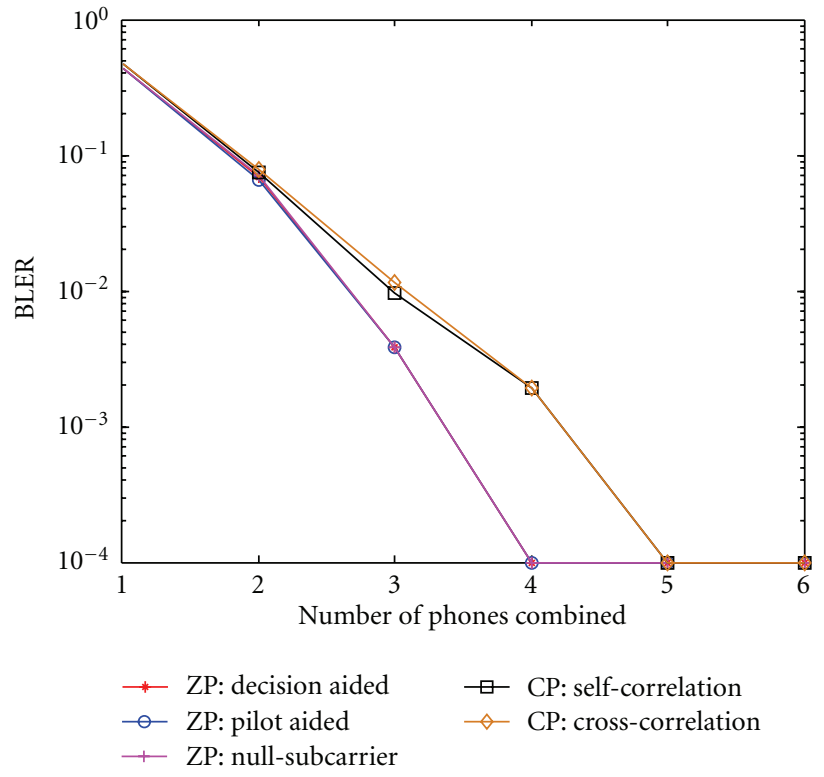

(a) Adding noise with power $\hat{\sigma}^{2}$

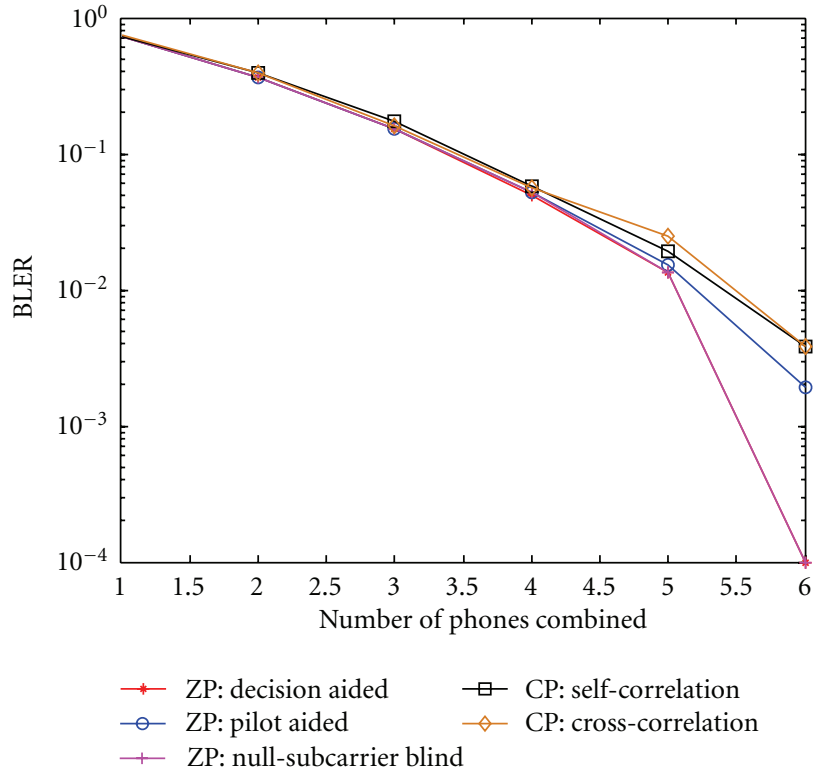

(b) Added noise with power $2 \widehat{\sigma}^{2}$

Figure 11: MACE10: BLER Performance using different Doppler estimation methods by adding artificial noise to the received signal, $\hat{\sigma}^{2}$ denoting the estimated ambient noise power.

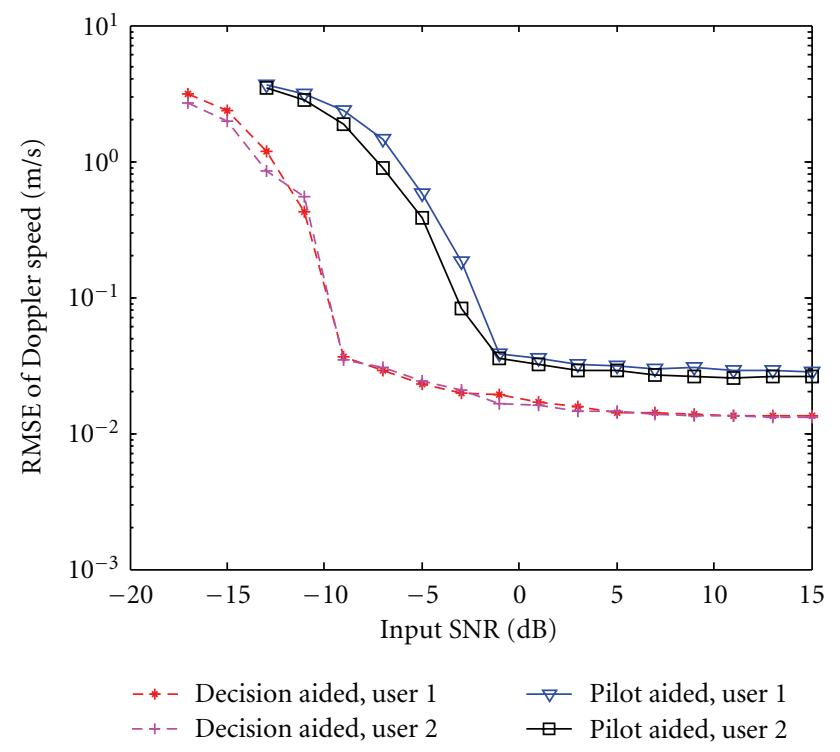

Figure 12: Pilot- and decision-aided Doppler scale estimation in a distributed two-user ZP-OFDM system.

noise to the recorded signal. The ground truth of the Doppler scale factor is not available. When computing the RMSE using (20) for each method, we use the estimated Doppler scale of the original file without adding the noise as the ground truth. Figure 10 shows the estimation performance of several approaches. Similar observations as the simulation results in Figures 3 and 5 are found.
(B) Test Case 2. In this test, we compare the BLER performance of an OFDM receiver where the resampling operation is carried out with different Doppler scale estimates from different methods.

Due to the relatively high SNR of the recorded signal, we create a semiexperimental data set by adding white Gaussian noise to the received signal. Define $\hat{\sigma}^{2}$ as the estimated ambient noise power in the original recorded signal. Figure 11 shows the BLER performance with different Doppler estimation approaches by adding different amount of noises to the received files.

One can see that the methods for ZP-OFDM outperform the methods for CP-OFDM, as the Doppler scale itself is continuously changing from block to block, as illustrated in Figure 8. Another interesting observation is that the null-subcarrier-based blind method has slight performance improvement relative to the pilot- and decision-aided methods. This agrees with the simulation results in Figure 5 that in the high SNR region, the blind estimation method does not suffer an error floor in the multipath channel, hence enjoys a better estimation performance.

\section{Extension to Distributed MIMO-OFDM}

If the transmitters in a multi-input multi-output (MIMO) system are co-located, the Doppler scales corresponding to all transmitters are similar, and hence a single-user blind Doppler scale estimation method would work well, as done in [10]. However, if the transmitters are distributed, for example in a system with multiple single-transmitter users, the Doppler scales for different users could be quite different, even with opposite signs [32]. We now investigate the performance of different Doppler scale estimation methods 


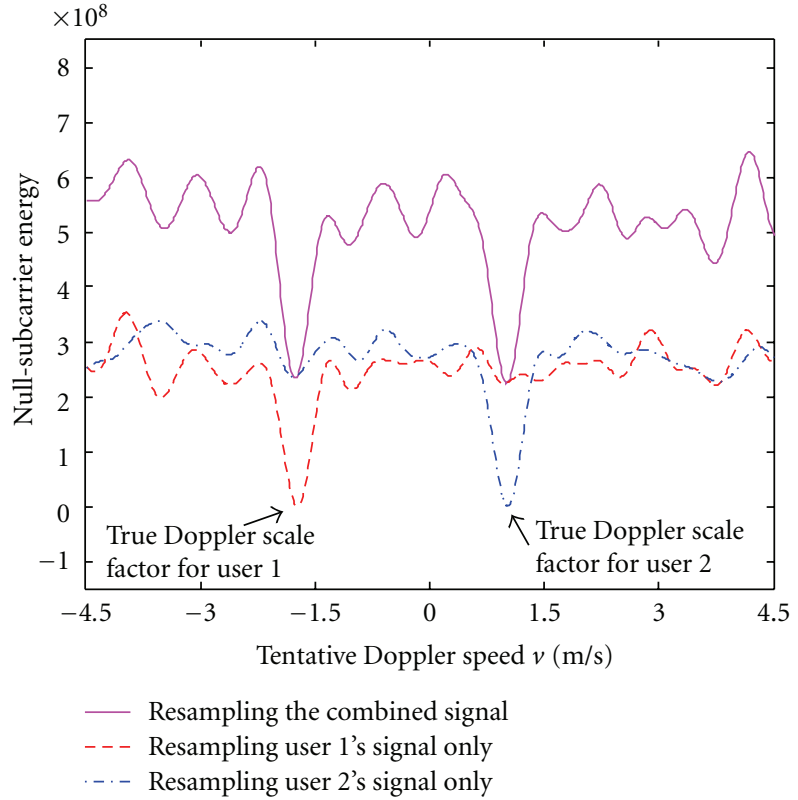

(a) Successful case

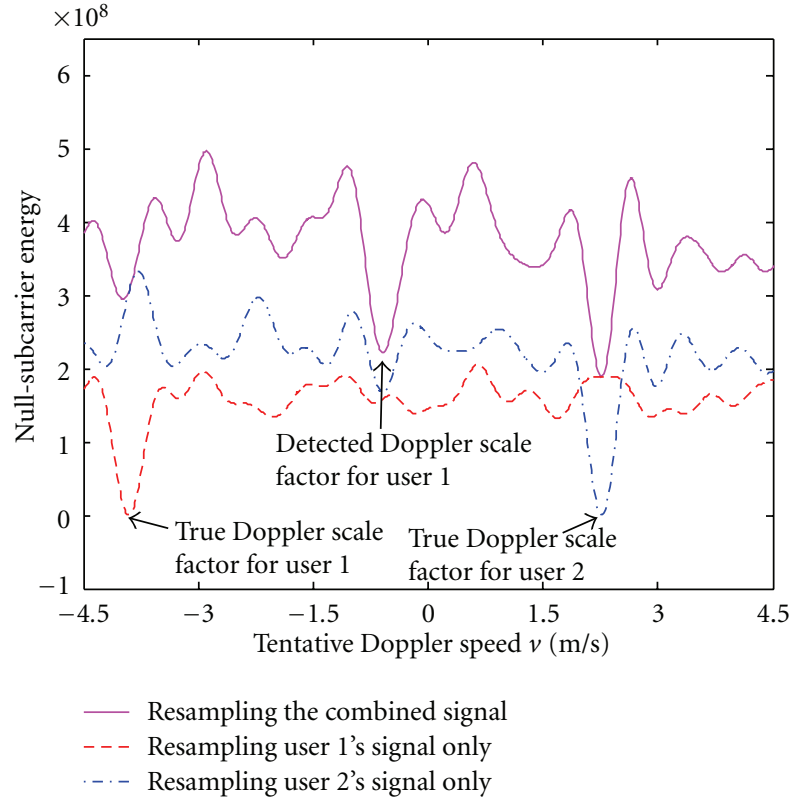

(b) Failed case

FIGURE 13: Illustration of the objective functions of the null-subcarrier-based method in a two-user system

in the presence of multiuser interference. We will use the ZPOFDM waveform as the reference design; similar conclusions can be applied to the CP-OFDM preamble. Only simulated data sets are used in the following tests.

6.1. Pilot- and Decision-Aided Estimation. We simulate a two-user system. Each user generates a multipath channel according to channel setting 2 independently. The positions of pilot, null, and data subcarriers are the same for different users. The pilot and data symbols of different users are randomly generated and hence are different.

Figure 12 depicts the RMSE performance of the pilotand decision-aided estimation methods. Compared with the performance in the single-user setting in Figure 5, there is performance degradation and the error floors are higher. However, both methods can achieve RMSE lower than $0.1 \mathrm{~m} / \mathrm{s}$ at low SNR values. Hence, both methods have robust performance in the presence of multiuser interference.

6.2. Null-Subcarrier-Based Blind Estimation. The null-subcarrier-based blind estimation method exploits the transmitted OFDM signal structure. Since all the users share the same positions of null subcarriers, there is a user-association problem even when multiple local minimums are found. We simulate a two-user system where the Doppler speeds of user 1 and user 2 are uniformly distributed within $[-4.5,-0.5] \mathrm{m} / \mathrm{s}$ and $[0.5,4.5] \mathrm{m} / \mathrm{s}$, respectively. Without adding the ambient noise to the received signal, Figure 13 demonstrates both successful and failed cases using the objective function in (12). The objective functions in the single-user settings are also included for comparison. One can see that the multiuser interference degrades the estimation performance significantly. Hence, although the blind method developed for the single user case can be used to colocated MIMOOFDM as in [10], it is not applicable to distributed MIMOOFDM where different users have different Doppler scales.

\section{Conclusion}

This paper compared different methods for Doppler scale estimation for a CP-OFDM preamble followed by ZP-OFDM data transmissions. Blind methods utilizing the underlying signalling structure work very well at medium to high SNR ranges, while cross-correlation-based methods can work at low SNR ranges based on the full or partial knowledge of the transmitted waveform. All of these methods are viable choices for practical OFDM receivers. In a distributed multiuser scenario, cross-correlation approaches are more robust against multiuser interference than blind methods.

\section{Acknowledgments}

This work is supported by the ONR Grant N00014-09-10704 (PECASE) and the NSF Grant ECCS-1128581. The authors thank Dr. Lee Freitag and his team for conducting the MACE10 experiment.

\section{References}

[1] I. F. Akyildiz, D. Pompili, and T. Melodia, "Challenges for efficient communication in underwater acoustic sensor networks," ACM SIGBED Review, vol. 1, no. 1, pp. 3-8, 2004.

[2] J.-H. Cui, J. Kong, M. Gerla, and S. Zhou, "The challenges of building scalable mobile underwater wireless sensor networks for aquatic applications," IEEE Network, vol. 20, no. 3, pp. 12$18,2006$. 
[3] M. Stojanovic, "Low complexity OFDM detector for underwater acoustic channels," in OCEANS 2006, usa, September 2006.

[4] B. Li, S. Zhou, M. Stojanovic, L. L. Freitag, and P. Willett, "Multicarrier communication over underwater acoustic channels with nonuniform Doppler shifts," IEEE Journal of Oceanic Engineering, vol. 33, no. 2, pp. 198-209, 2008.

[5] F. Qu and L. Yang, "Basis expansion model for underwater acoustic channels?” in OCEANS 2008, can, September 2008.

[6] T. Kang and R. A. Iltis, "Iterative carrier frequency offset and channel estimation for underwater acoustic OFDM systems," IEEE Journal on Selected Areas in Communications, vol. 26, no. 9, Article ID 4686804, pp. 1650-1661, 2008.

[7] G. Leus and P. A. Van Walree, "Multiband OFDM for covert acoustic communications," IEEE Journal on Selected Areas in Communications, vol. 26, no. 9, Article ID 4686805, pp. 1662$1673,2008$.

[8] P. A. V. Walree and G. Leus, "Robust underwater telemetry with adaptive turbo multiband equalization," IEEE Journal of Oceanic Engineering, vol. 34, no. 4, Article ID 5299204, pp. 645-655, 2009.

[9] A. Abdi and H. Guo, "A new compact multichannel receiver for underwater wireless communication networks," IEEE Transactions on Wireless Communications, vol. 8, no. 7, pp. 3326-3329, 2009.

[10] B. Li, J. Huang, S. Zhou et al., "MIMO-OFDM for highrate underwater acoustic communications," IEEE Journal of Oceanic Engineering, vol. 34, no. 4, Article ID 5290161, pp. 634-644, 2009.

[11] C. R. Berger, S. Zhou, J. C. Preisig, and P. Willett, "Sparse channel estimation for multicarrier underwater acoustic communication: from subspace methods to compressed sensing," IEEE Transactions on Signal Processing, vol. 58, no. 3, pp. 1708 1721, 2010.

[12] P. Ceballos and M. Stojanovic, "Adaptive channel estimation and data detection for underwater acoustic MIMO-OFDM Systems," IEEE Journal of Oceanic Engineering, vol. 35, no. 3, Article ID 5530330, pp. 635-646, 2010.

[13] T. Kang, H. C. Song, W. S. Hodgkiss, and J. Soo Kim, "Longrange multi-carrier acoustic communications in shallow water based on iterative sparse channel estimation," Journal of the Acoustical Society of America, vol. 128, no. 6, pp. EL372-EL377, 2010.

[14] J. Tao, Y. R. Zheng, C. Xiao, and T. C. Yang, "Robust MIMO underwater acoustic communications using turbo block decision-feedback equalization," IEEE Journal of Oceanic Engineering, vol. 35, no. 4, Article ID 5634060, pp. 948-960, 2010.

[15] J. Tao, J. Wu, Y. R. Zheng, and C. Xiao, "Enhanced MIMO LMMSE turbo equalization: algorithm, simulations, and undersea experimental results," IEEE Transactions on Signal Processing, vol. 59, no. 8, Article ID 5756490, pp. 3813-3823, 2011.

[16] K. Tu, D. Fertonani, T. M. Duman, M. Stojanovic, J. G. Proakis, and P. Hursky, "Mitigation of intercarrier interference for OFDM over time-varying underwater acoustic channels," IEEE Journal of Oceanic Engineering, vol. 36, no. 2, Article ID 5766772, pp. 156-171, 2011.

[17] J.-Z. Huang, S. Zhou, J. Huang, C. R. Berger, and P. Willett, "Progressive inter-carrier interference equalization for OFDM transmission over time-varying underwater acoustic channels," IEEE Journal on Selected Topics in Signal Processing, vol. 5, no. 8, Article ID 5893908, pp. 1524-1536, 2011.

[18] H. Wan, R.-R. Chen, J. W. Choi, A. C. Singer, J. C. Preisig, and B. Farhang-Boroujeny, "Markov chain Monte Carlo detection for frequency-selective channels using list channel estimates," IEEE Journal on Selected Topics in Signal Processing, vol. 5, no. 8, Article ID 6053992, pp. 1537-1547, 2011.

[19] A. Song, M. Badiey, V. McDonald, and T. Yang, "Time reversal receivers for high rate multiple-input/multiple-output communication," IEEE Journal of Oceanic Engineering, vol. 34, no. 4, pp. 525-538, 2011.

[20] B. S. Sharif, J. Neasham, O. R. Hinton, and A. E. Adams, "Computationally efficient Doppler compensation system for underwater acoustic communications," IEEE Journal of Oceanic Engineering, vol. 25, no. 1, pp. 52-61, 2000.

[21] S. Yerramalli and U. Mitra, "Optimal resampling of OFDMm signals for multiscale-multilag underwater acoustic channels," IEEE Journal of Oceanic Engineering, vol. 36, no. 1, Article ID 5732766, pp. 126-138, 2011.

[22] J. R. Klauder, A. C. Price, S. Darlington, and W. J. Albersheim, "The theory and design of chirp radars," Bell System Technical Journal, vol. 39, pp. 745-808, 1960.

[23] S. Kramer, "Doppler and acceleration tolerances of high-gain, wideband linear FM correlation sonars," Proceedings of the IEEE, vol. 55, no. 5, pp. 627-636, 1967.

[24] J. J. Kroszczyński, "Pulse compression by means of linearperiod modulation," Proceedings of the IEEE, vol. 57, no. 7, pp. 1260-1266, 1969.

[25] T. Yang, "Underwater telemetry method using Doppler compensation," U.S. Patent 6512720, 2003.

[26] J. P. Costas, "A study of a class of detection waveforms having nearly ideal range-Doppler ambiguity properties," Proceedings of the IEEE, vol. 72, no. 8, pp. 996-1009, 1984.

[27] J. G. Proakis, Digital Communications, McGraw-Hill, New York, NY, USA, 4th edition, 2001.

[28] R. Frank, S. Zadoff, and R. Heimiller, "Phase shift pulse codes with good periodic correlation properties (corresp.)," IRE Transactions on Information Theory, vol. 8, no. 6, pp. 381-382, 1962.

[29] S. F. Mason, C. R. Berger, S. Zhou, and P. Willett, "Detection, synchronization, and doppler scale estimation with multicarrier waveforms in underwater acoustic communication," IEEE Journal on Selected Areas in Communications, vol. 26, no. 9, Article ID 4686803, pp. 1638-1649, 2008.

[30] H. Yan, L. Wan, S. Zhou et al., "DSP based receiver implementation for OFDM acoustic modems," Physical Communication, vol. 5, no. 1, pp. 22-32, 2012.

[31] X. Ma, C. Tepedelenlioğlu, G. B. Giannakis, and S. Barbarossa, "Non-data-aided carrier offset estimators for OFDM with null subcarriers: identifiability, algorithms, and performance," IEEE Journal on Selected Areas in Communications, vol. 19, no. 12, pp. 2504-2515, 2001.

[32] K. Tu, T. M. Duman, J. G. Proakis, and M. Stojanovic, "Cooperative MIMO-OFDM communications: receiver design for Doppler-distorted underwater acoustic channels," in Proceedings of the 44th Asilomar Conference on Signals, Systems and Computers (Asilomar '10), pp. 1335-1339, Pacific Grove, Calif, USA, November 2010.

[33] J. Huang, S. Zhou, and P. Willett, "Nonbinary LDPC coding for multicarrier underwater acoustic communication," IEEE Journal on Selected Areas in Communications, vol. 26, no. 9, Article ID 4686807, pp. 1684-1696, 2008. 
[34] B. Friedlander, "On the Cramer- Rao bound for time delay and Doppler estimation (corresp.)," IEEE Transactions on Information Theory, vol. 30, no. 3, pp. 575-580, 1984.

[35] X. X. Niu, "Wavelet based approach for joint time delay and doppler stretch measurements," IEEE Transactions on Aerospace and Electronic Systems, vol. 35, no. 3, pp. 1111-1119, 1999. 

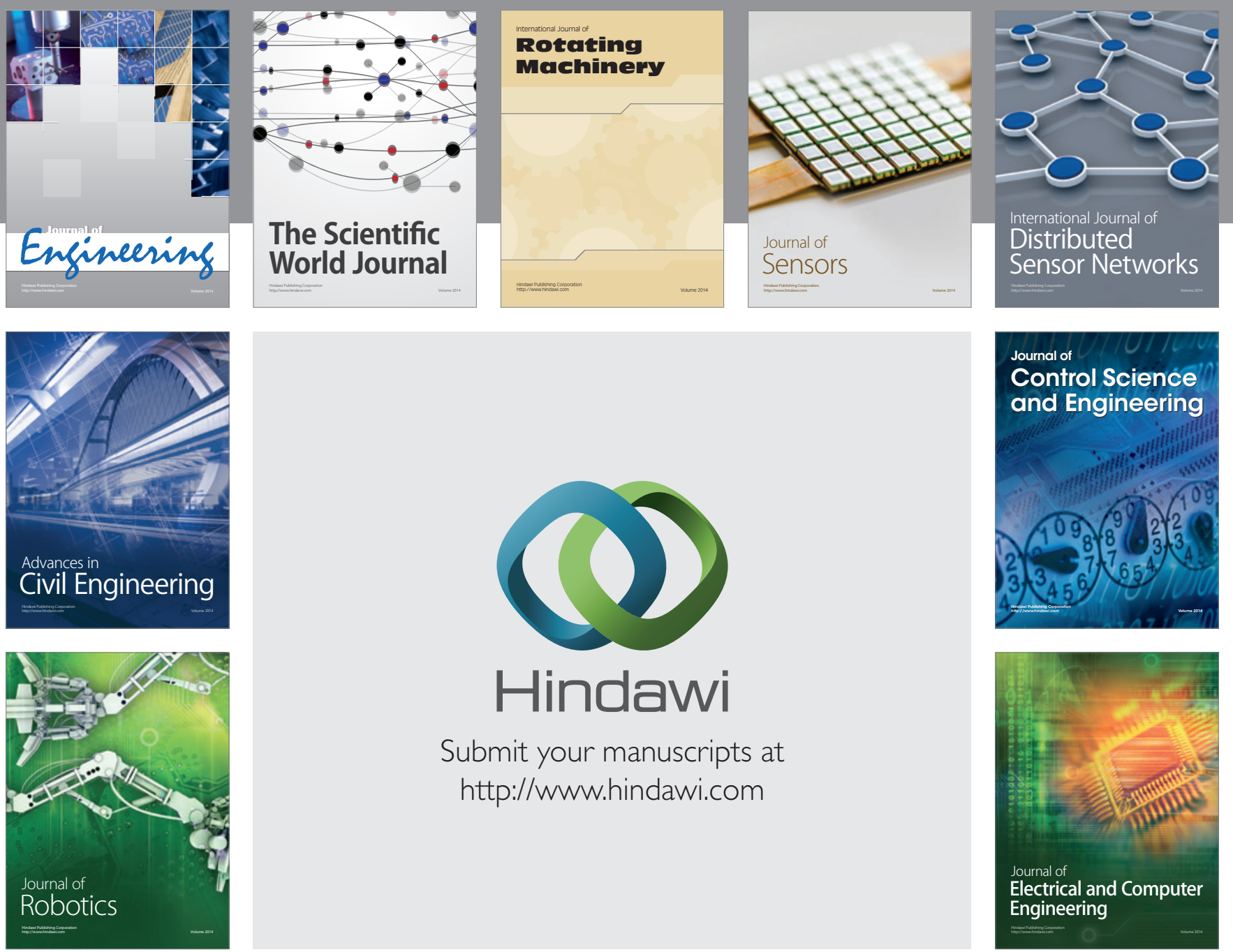

Submit your manuscripts at

http://www.hindawi.com
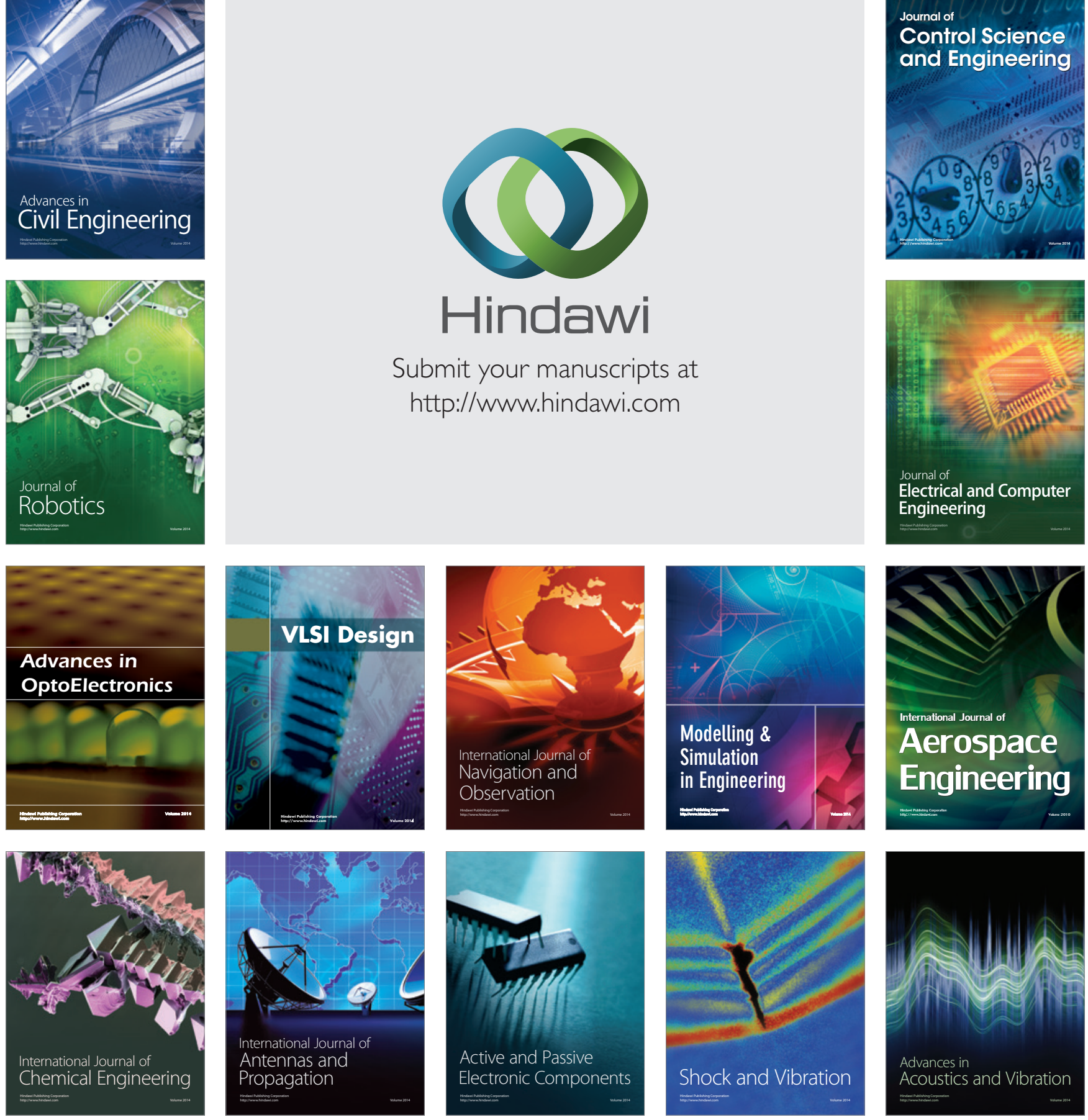